\title{
Study of R-161 refrigerant as an Alternate Refrigerant to various other refrigerants
}

\author{
Kalpesh N. Kothale ${ }^{\dagger *}$, Kundlik V. Mali ${ }^{\dagger}$ and S. D. Nimbalkar ${ }^{\dagger}$ \\ ${ }^{\dagger}$ Mechanical Engineering Department, MAEER's MIT College of Engineering, Pune \\ Accepted 03 March 2016, Available online 15 March 2016, Special Issue-4 (March 2016)
}

\begin{abstract}
This paper discusses the suitability of R-161 refrigerant over other refrigerants. Apart from having zero ODP and a very low GWP value of $12, R-161$ refrigerant can prove a promising alternative to existing alternate refrigerant and various other HCFCs facing the fate of phase out. The paper discusses various experimental results obtained in different research and discusses them on the basis of COP, discharge temperature, Cooling capacity and Power consumption. Five different research works were studied to make a firm comment on the effect of use of $R-161$ on the above mentioned parameters. The general finding show that $R$-161 refrigerant give better COP, increased refrigeration effects and lower discharge temperatures. Power consumed by use of $R-161$ was found to be greater than other refrigerants. In order to suppress the flammability property of R-161, some research done with a mixture of $R-161$ \& other refrigerants, with same thermodynamic property was also included in this study. Similar results were obtained from them.
\end{abstract}

Keywords: Alternate refrigerants, R-161, GWP, COP, Refrigeration Effect, Discharge Temperature, Power consumption.

\section{Introduction}

Till the end of last century, rapid developments in the world resulted in increased refrigeration \& airconditioning demands. The increased uncontrolled use of CFC and other ODS had adversely affected the environment in the form of Ozone layer depletion. Concerning this problem, CFCs and HCFCs refrigerants have been banned in developed countries and gradually phased out in developing countries as required by the Montreal Protocol (1987) and following amendments.

India became a party to the Montreal Protocol in 1992 and had ratified several amendments. As per the protocols, various HCFC refrigerants are being phased out. The rapid phase out various $\mathrm{CFC}$ and HCFC refrigerants due to high GWP values has made engineers to turn to new refrigerants. As per the new regulatory policy, a refrigerant is required to have zero ODP and a low GWP value. The alternate refrigerants currently in use have lower or zero ODP, but a higher GWP values.

In such scenario, ethyl fluoride or R-161 with zero ODP and a very low GWP of 12 , is gaining rapid importance. Not in use, till recently, due to its inflammable nature, it is now proved that along with

*Corresponding author: Kalpesh N. Kothale inhibitors, R-161 can prove to be an important alternate Refrigerant. The present paper discusses various theoretical \& experimental results obtained using R-161 refrigerant over commonly used refrigerants. The results obtained with R-161 refrigerant show increased COP of the system, reduced discharge temperature etc. over the commonly used refrigerants..

This serves as inspiration for presenting a review paper on this topic. This paper particularly discusses the suitability of R-161 refrigerant over commonly used refrigerants with higher GWP. R-161 refrigerant has not been seriously considered as an alternate refrigerant due to its flammability and toxicity. Although, the latter has been disproved by recent research works, thus R-161 is getting a rapid attention as an alternate refrigerant with zero ODP and very less GWP.

\section{Literature review}

Yongmei Xuan \& Guangming Chen proposed R-161 as an alternate refrigerant for R-502 in their research They used a ternary near azeotropic mixture of R-161 with thermodynamic properties similar to that of R502. The refrigerant $\mathrm{R}-502$ then facing a fate of complete phase out and thus the performance were compared with R-404A, a main alternate refrigerant to $\mathrm{R}-502$. They found out that under lower evaporating 
conditions, the COP of both the refrigerants were almost same. While, at higher evaporating temperature, R-161 mixture show an increased COP than R-404A.

Xiao-hong Han et al. presented R-161 as a better alternative refrigerant to HFC-410a, over HFC-32. The theoretical, as well as experimental analysis on a vapor compression cycle with rotary compressor showed that the COP of R-161 was found higher by $>15 \%$ than that of HFC-32 and HFC-410a. Also, the discharge temperature was found to be lower for HFC-161. The cooling capacity and power consumption of HFC-161 were reported lowest among the other three.

Yingwen Wu et al. in their feasibility study of R-161, R22 and R-290 for a $3.5 \mathrm{~kW}$ heat pump air conditioner showed that R-161 has better thermodynamic performance than R-290. Their simulation results showed that EER of R-161 increased by $2 \%$ and a reduced cooling capacity compared to $\mathrm{R}-22$. It further reports in a declined discharge temperature and reduced refrigerant charge.

Recently, M. Wu et al developed theoretical analysis and experiments to check feasibility of HFC-161 as an alternative refrigerant for HFC-134a. For evaporation temperature range of $-5^{\circ} \mathrm{C}$ to $5^{\circ} \mathrm{C}$ and condensation temperature $50^{\circ} \mathrm{C}$ to $65^{\circ} \mathrm{C}$, theoretical results show increased refrigerating effect for HFC-161 than HFC134a. The COPs of HFC-161 were higher than that of HFC-134a at evaporation temperatures of $-5^{\circ} \mathrm{C}, 0^{\circ} \mathrm{C}$, $5^{\circ} \mathrm{C}$. The refrigeration capacity and compressor power consumption of HFC-161 were about $56 \%$ and $47 \%$ higher than those of HFC-134a.

Subodh Deshpande \& D. N. Hatkar et. al proposed a new refrigerant using a $(60 / 40 \%$ by wt) combination of R-134A and R-161 for domestic refrigerators. As per the theoretical performance assessment, the combination provided a COP similar to that obtained by R-134A. For same COP the specific and volumetric Refrigeration effect were found to be increased by $37 \%$ and $32.6 \%$, respectively. The reported GWP value of the combination mixture was reported lower than that of R-134A.

\section{Physical Characteristics of R-161}

Table lists down the general properties of R-161 refrigerant.

Table 1 Basic Thermodynamic properties of R-161

\begin{tabular}{|l|l|l|}
\hline Properties & Unit & R161 \\
\hline Molecular & $\mathrm{g} / \mathrm{mol}$ & 48.06 \\
\hline Normal boiling point & ${ }^{\circ} \mathrm{C}$ & -37.6 \\
\hline Critical temperature & ${ }^{\circ} \mathrm{C}$ & 102.2 \\
\hline Critical pressure & $\mathrm{MPa}$ & 5.09 \\
\hline Liquid state density $\left(25^{\circ} \mathrm{C}\right)$ & $\mathrm{kg} / \mathrm{m}^{3}$ & 644 \\
\hline Ozone depletion potential & - & 0 \\
\hline GWP $_{100}$ & - & 12 \\
\hline Atmospheric life time & $\mathrm{a}$ & 0.21 \\
\hline Lower flaming limit by mass & $\mathrm{kg} / \mathrm{m}^{3}$ & 0.075 \\
\hline Lower flaming limit by volume & $\%$ & 3.8 \\
\hline Burning velocity & $\mathrm{cm} / \mathrm{s}$ & 38.3 \\
\hline Combustion heat & $\mathrm{MJ} / \mathrm{kg}$ & 26.6 \\
\hline Occupational exposure limit & $\mathrm{ppm}$ & 1800 \\
\hline Safety Class & & $\mathrm{A} 3$ \\
\hline
\end{tabular}

Apart from having zero ODP and a very lower value of GWP, the reason for selecting R-161 as alternate refrigerant is its characteristic similarity to various refrigerants in consideration. It can be seen clearly from the saturation pressure vs. temperature graph.

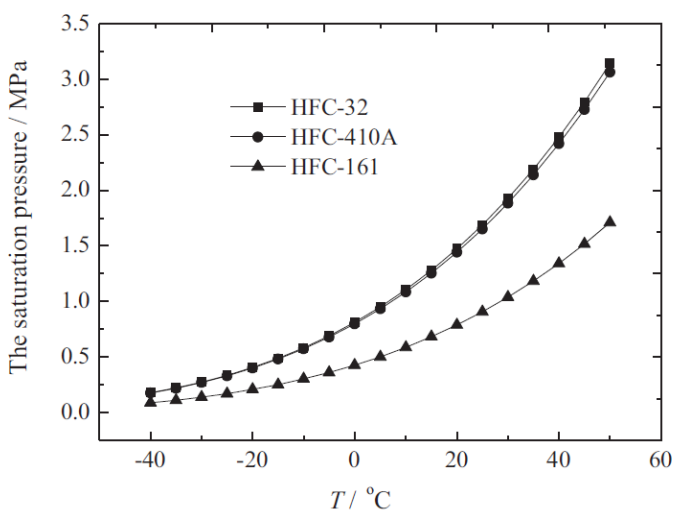

Fig.1 Saturation Temperature Vs. pressure curve of R-32, R-410A, R-161

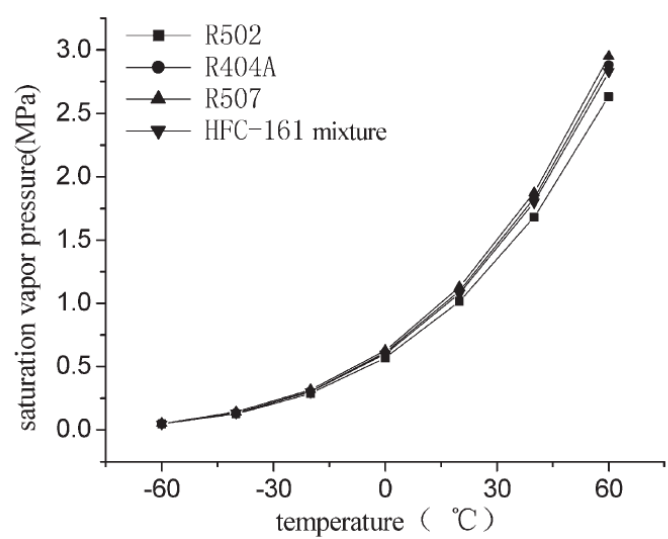

Fig.2 Saturation Temperature Vs. pressure curve of

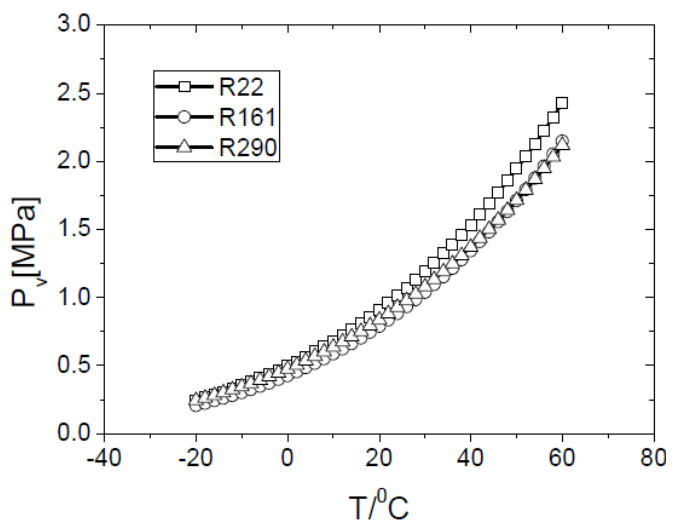

Fig.3 Saturation Temperature Vs. pressure curve of R-22, R-161, R290

Figure (a) shows the saturation curve for R-161, R-32, and HFC-410A. The lower saturation pressure line for R-161 suggests that the system using the other two refrigerants can be easily modified at low cost. 


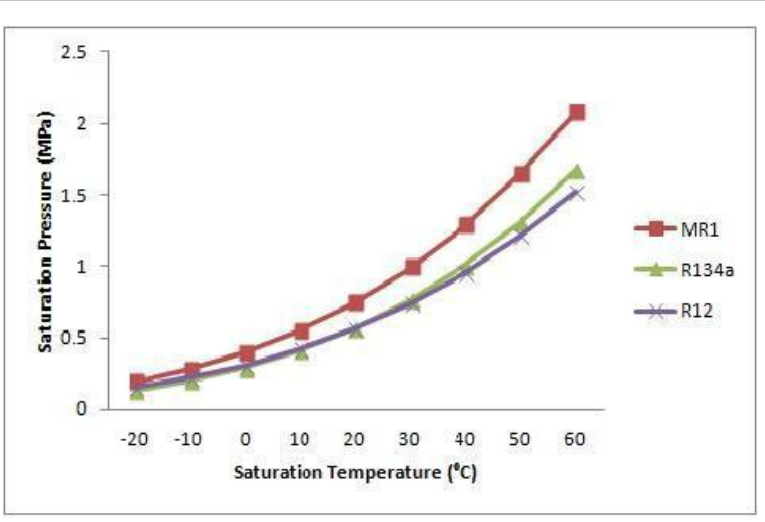

Fig.4 Saturation Temperature Vs. pressure curve of R-134A, R-12, MR1(R-161+R-134A)

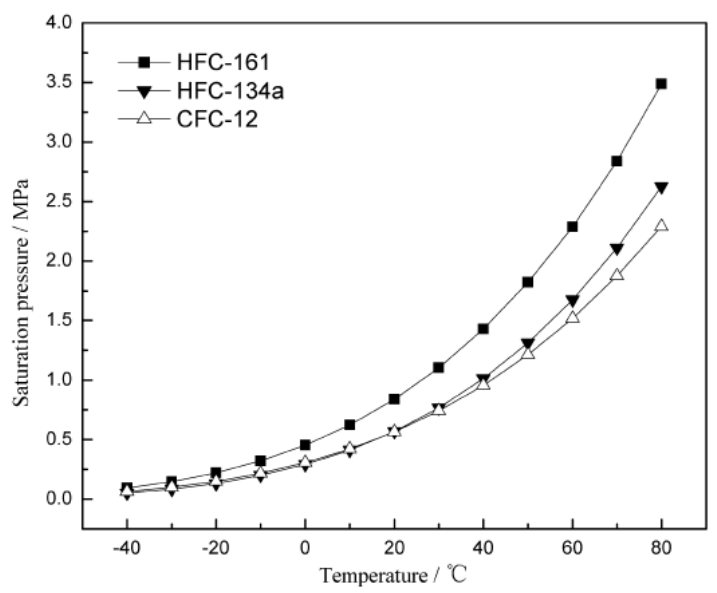

Fig.5 Saturation Temperature Vs. pressure curve of R161, R-134A, CFC-12.

Similarly, Fig.(b),(c),(d) and (e) show saturation pressure diagram of R-161 and other refrigerants to be substituted by it. It can be easily concluded by looking at the curves, that $\mathrm{R}-161$ resembles many properties of the old refrigerants.

\section{Experimental Setup}

The experimental setup commonly used in all the research work is similar to the one developed by Xiaohang Han et. al to test R-161 refrigerant as an alternative refrigerant to R-410A. The setup is of vapor compression refrigeration cycle. It is composed of a condenser, a subcooler, the throttle valve, compressor, an evaporator fitted in a calorimeter, some measuring devices. The setup uses two type of refrigerant: the one being tested and the other refrigerant is used in the calorimeter. A heater, submerged under the other refrigerant is used to heat the refrigerant. The evaporated refrigerant moves up to the evaporator coils and exchanges heat with the main refrigerant flowing through it. The pressure in the condenser and evaporator is maintained by controlling the mass flow rate of fluids to the respective parts. A subcooler is also used. A receiver or similar arrangement is made to ensure only liquid flows through to the expansion device. Various manometers and pressure gauges are installed to meter the readings around the cycle.

The readings are taken for various working conditions. The conditions are created by properly adjusting the flow rate to the condenser and subcooler and controlling the heat input to the calorimeter. At least four readings were taken, in order to ensure the repeatability of the Test rig. Readings were averaged over, in order to get the most accuracy.

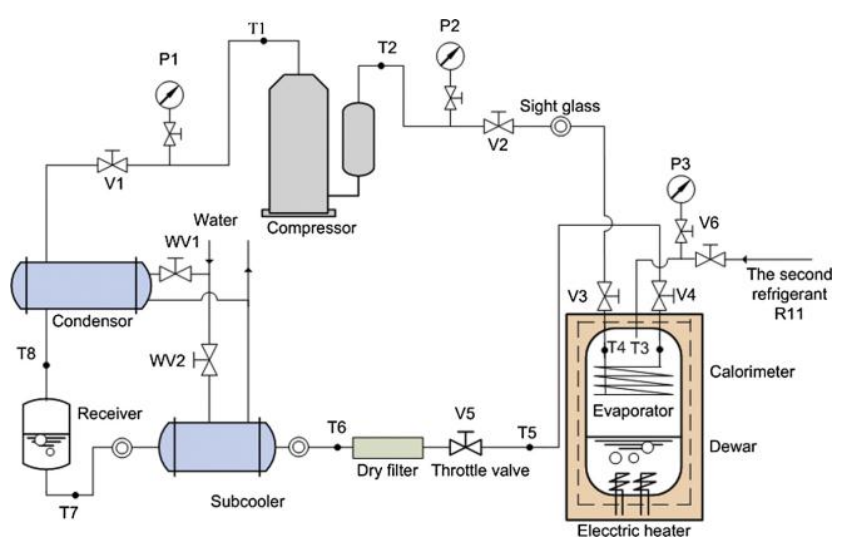

Fig.6 The Schematic of Experimental Apparatus

\section{Results \& Discussion}

The experimental results obtained by testing R-161 refrigerant and other refrigerants under different working conditions were obtained in various studies. These results will be discussed in detail through following sections. The parameters discussed are
(a) Coefficient of Performance;
(b) Discharge Temperature;
(c) Refrigeration Capacity;
(d) Power Consumption.

(a) Coefficient of Performance, COP

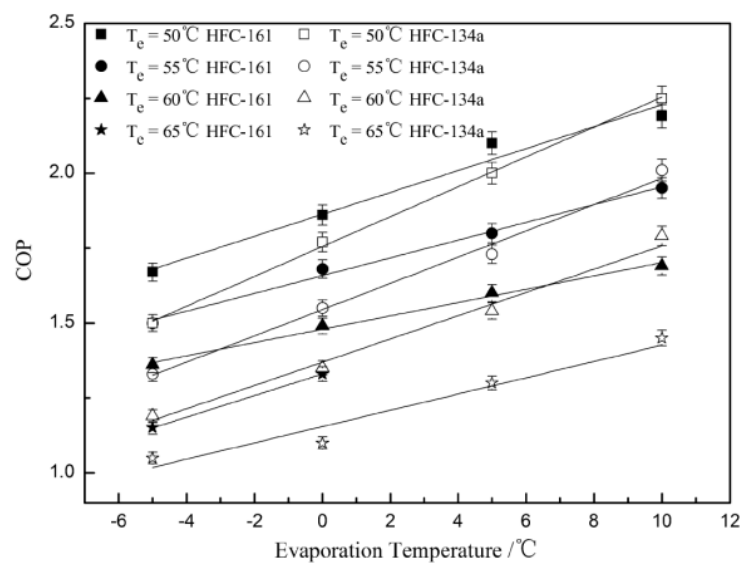

Fig.7 COPs of HFC-161 and HFC-134a at various working conditions in experiments 


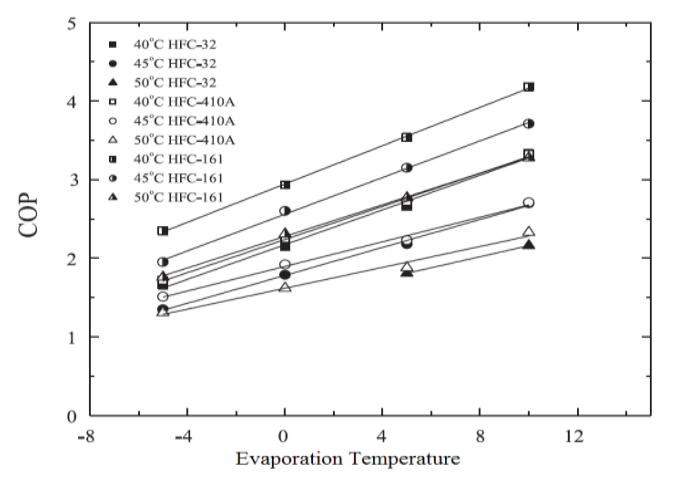

Fig.8 COPs of HFC-32, HFC-410A \& HFC-161 at various working conditions in experiments

Fig.(7-9) shows the variation of COP for different evaporation temperature is clear that the COP values for HFC-161 is higher than that of HFC-134a. As the evaporation temperature goes on increasing, the COP is almost increased by $30-40 \%$, but above $8^{\circ} \mathrm{C}$ the COP of HFC-134a overtakes the HFC-161 values. For the test between HFC-161, HFC-32 \& HFC-410A, the COP of R161 refrigerant was found to be the highest, followed by HFC-410A and HFC-32, respectively.

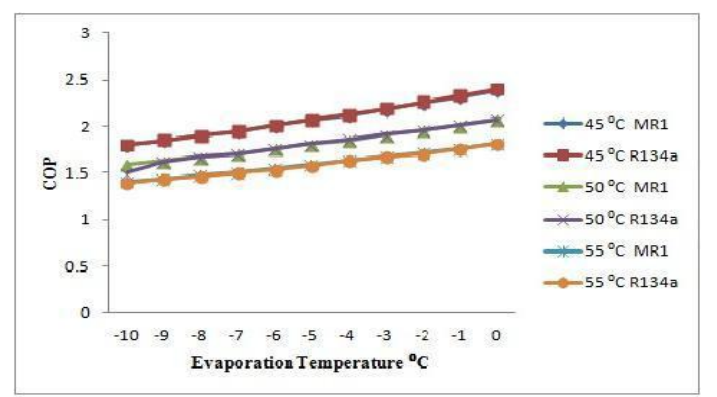

Fig. 9 COPs of R-134a and MR1 (R-134a + R-161) at various working conditions in experiments.

A combination of R-134A and R-161 was found to have same COP as that due to only R-134A. The GWP value of this combination is less than that of pure R-134A. The COP is found to be same for a wide range of temperatures, as can be seen from the fig.

\section{(b) Discharge Temperature}

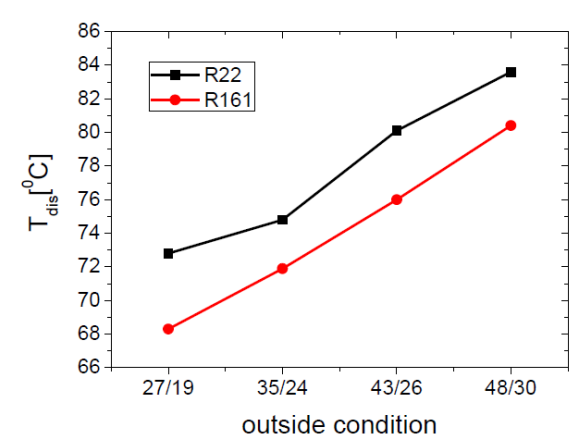

Fig.10 Discharge Temperatures of R-161 and R-22 at various working conditions in experiments

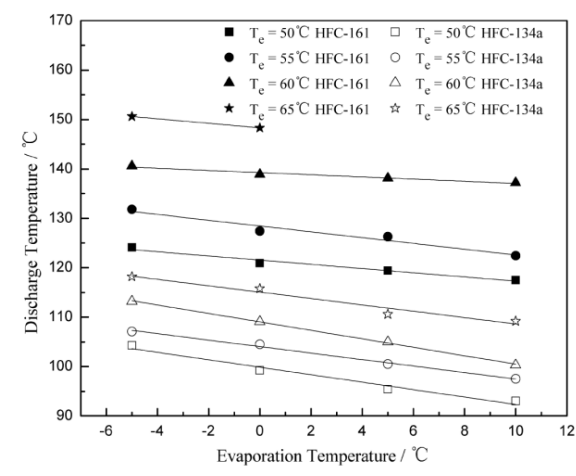

Fig.11 Discharge Temperatures of HFC-161 and HFC-134a at various working conditions in experiments

As seen in fig.10 the compressor discharge temperature in the comparison test between R-161 and R-22, was found to be lower for R-161 than the latter, in cooling mode. Although, there is a rise in discharge temperature with the rise in outside conditions, R-161 shows lower discharge temperature over the range. In case of R-161mixture and R-404A, the discharge temperature was found to be more for R161 refrigerant at lower evaporator temperatures $\left(\mathrm{T}_{\mathrm{e}}=-\right.$ $\left.40^{\circ} \mathrm{C}\right)$, but at higher evaporator temperature $\left(\mathrm{T}_{\mathrm{e}}=-\right.$ $40^{\circ} \mathrm{C}$ ) , R-161 showed lower discharge temperature than R-404A.

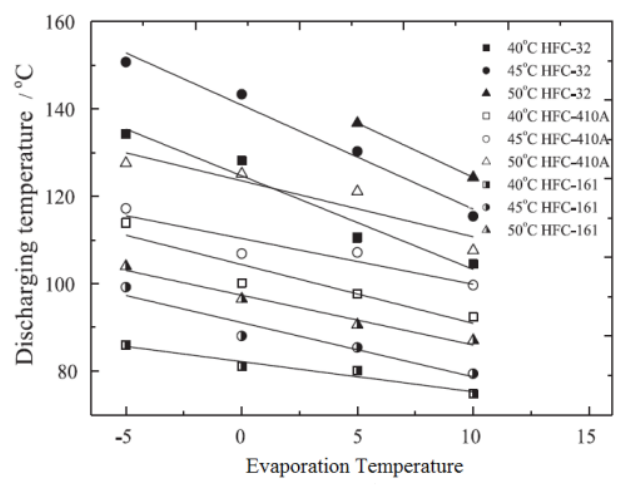

Fig.12 Discharge Temperatures of HFC-32, HFC-410A and HFC-161 at various working conditions in experiments

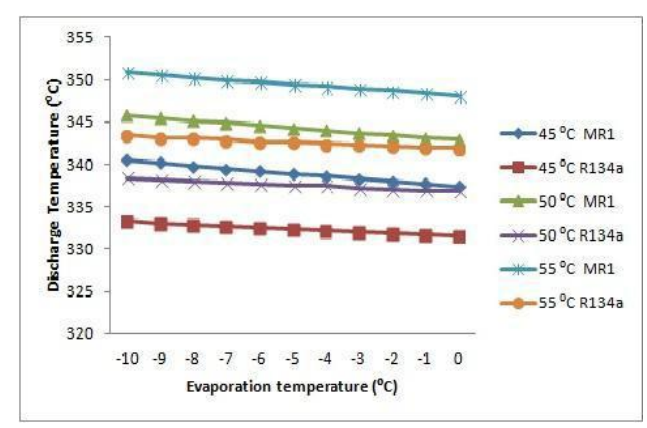

Fig.13 Discharge Temperatures of R-134a and MR1 (R$134 \mathrm{a}+\mathrm{R}-161$ ) at various working conditions in experiments 
In the performance study of R-161 as a replacement over R-32, potential replacement over R-410A, discharge temperature was reported to be lowest for R-161 out of the three. The trend was observed to be followed over a evaporation temperature range of $-5^{\circ} \mathrm{C}$ to $10^{\circ} \mathrm{C}$. Similar results were obtained in the experimental tests between R-134A and R-161 (Fig.11). The mixture of $\mathrm{R}-161$ and $\mathrm{R}-134 \mathrm{~A}$ reported a higher discharge temperature over R-134A, in the tests carried for domestic refrigeration.

\section{(c) Refrigeration Capacity}

In the theoretical performance study for refrigeration capacity using R-161, R-22 and its potential replacement R-290, the cooling capacity was calculated as intermediate between that of R-22 and R-290.

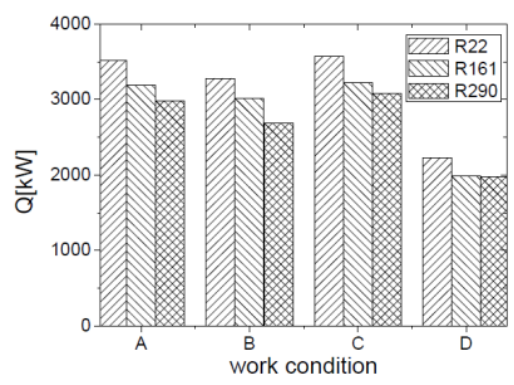

Fig.14 Theoretical Refrigeration capacities of R-22 and $\mathrm{R}-161$ at various working conditions

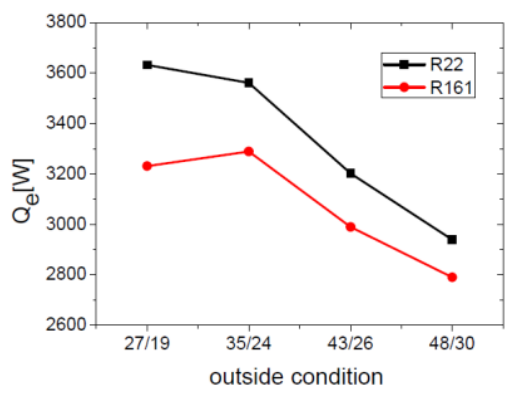

Fig.15 Refrigeration capacities of R-22 and R-161 at various working conditions in experiments

The experimental findings confirm to this calculations, as can be seen from fig.14 and fig.15. The cooling capacity of R-161 was found better than the replacement R-290 for phase out R-22.

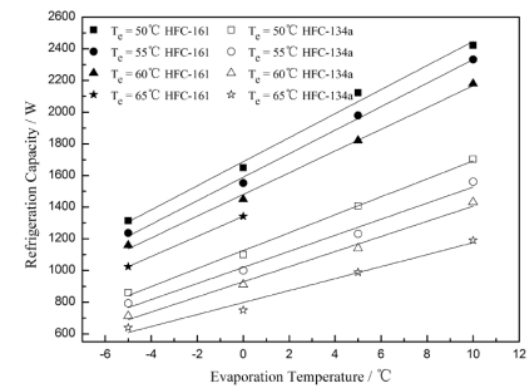

Fig.16 Refrigeration capacities of HFC-134a and HFC161 at various working conditions in experiments

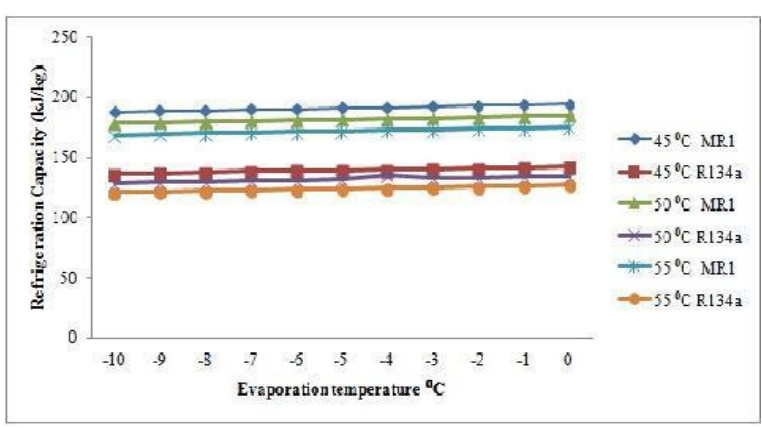

Fig.17 Refrigeration capacities of R-134a and MR1 (R-134a + R-161) at various working conditions in experiments

Fig.16 shows the variation of refrigeration capacities of R-161 and R-134A. The refrigeration capacity was found to be less than that of R-134A. But, the R-161/R$134 \mathrm{~A}$ mixture show better refrigeration capacity than R-134A (Fig.17)

(d) Power Consumption

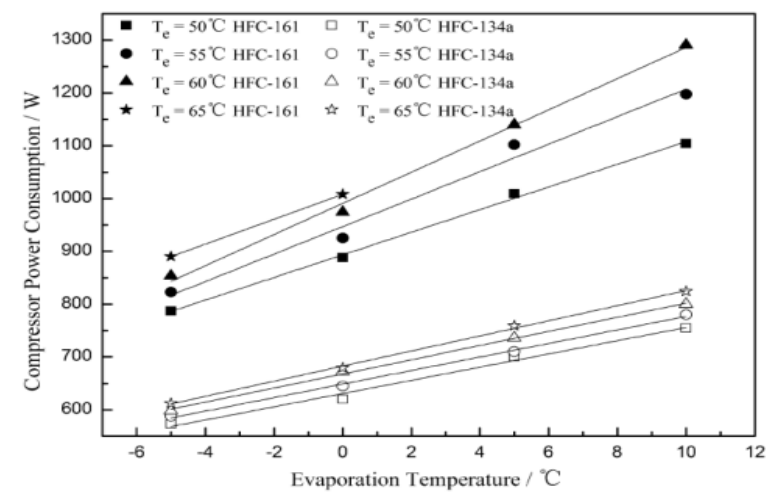

Fig.18 Power consumption for HFC-161 \& HFC-134a at various working conditions in experiments

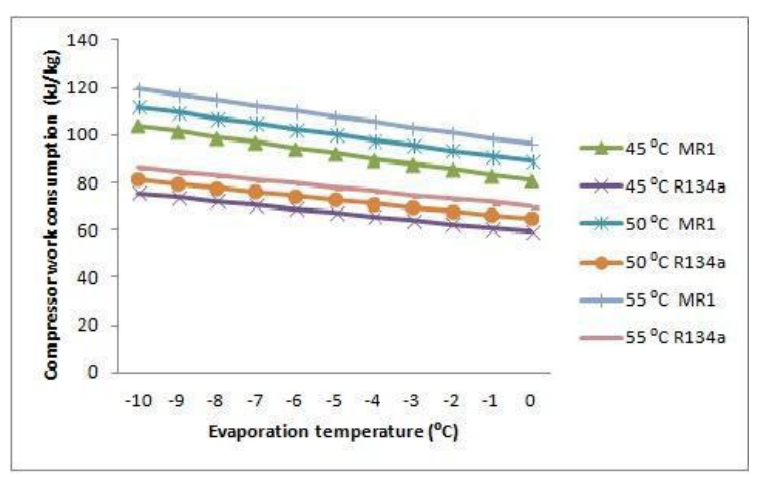

Fig.19 Power consumption for R-134a and MR1 (R$134 \mathrm{a}+\mathrm{R}-161$ ) at various working conditions in experiments

As seen from the two figures above, the power consumption by R-161 was reported to be higher than the other refrigerants. But, In comparison to R-32 and R-410A, R-161 show a very less power consumption over a wide evaporation temperature range. 


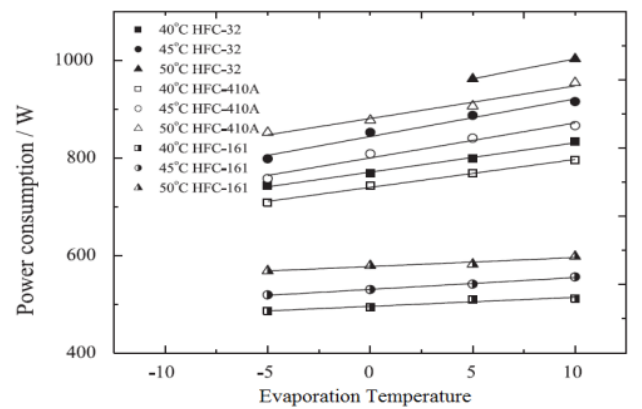

Fig.20 Power consumption for HFC-32, HFC-410A, HFC-161 at various working conditions in experiments

\section{Conclusion}

Based on the various theoretical studies and experimental research works, It can be concluded that R-161 with better thermodynamic and thermo physical properties can serve as a promising alternative over various refrigerants. It can be used successfully as a mixture, so as to suppress its inflammable nature. The zero ODP value and lower GWP value of R-161 makes it an attractive choice in the list of fourth generation refrigerants. It can be concluded that use of R-161 refrigerant gives an overall increased COP for the system. In spite of all these advantages, a very few experimental research has been reported so far. Thus, there is a lot scope for experimental research work on application of R-161 refrigerant as an alternate refrigerant.

\section{Acknowledgement}

The author of this paper would like to thank Dr. K. V. Mali, (Associate Professor, MAEERs MIT College of Engineering, Pune) and Prof. S. D. Nimbalkar (Assistant Professor, MAEERs MIT College of Engineering, Pune) for their constant encouragement and guidance, in this review work.

\section{References}

Xiao-hong Han, Yu Qiu, Peng Li, Ying-jie Xu, Qin Wang, Guangming Chen, Cycle performance studies on HFC-161 in a small-scale refrigeration system as an alternative refrigerant to HFC-410A, Energy and Buildings 44 (2012) 33-38,

doi:10.1016/j.enbuild.2011.10.004

Yongmei Xuan, Guangming Chen, Experimental study on HFC-161 mixture as an Alternative Refrigerant to R502.

doi:10.1016/j.ijrefrig.2004.04.003

Yingwen Wu, Xiangfei Liang, Xiaoping Tu, Rong Zhuang, Study of R161 Refrigerant for Residential Air conditioning Applications, International Refrigeration and Air Conditioning Conference at Purdue, July 16-19, 2012; paper 1189.

http://docs.lib.purdue.edu/iracc/1189

M. Wu, X.R. Yuan, Y.J. Xu, X.G. Qiao, X.H. Han, G.M. Chen, Cycle performance study of Ethyl Fluoride in the Refrigeration System of HFC-134a, Applied Energy 136 (2014), Page No. 1004-1009.

http://dx.doi.org/10.1016/j.apenergy.2014.05.068

Subodh Deshpande, D. N. Hatkar, Study of Performance of Binary Mixture of R134a

and R161 as a Substitution of R134a in a Domestic Refrigerator, International Journal of Engineering Research \& Technology (IJERT), Vol. 4 Issue 06, June2015.

ISSN: 2278-0181 [6] X.H. Han, Q. Wang, Z.W. Zhu, G.M. Chen, Cycle performance study on R32/R125/R161 as an alternative refrigerant to R407C. Applied Thermal Engineering 27 (2007), Page No. 2559-2565.

doi:10.1016/j.applthermaleng.2007.01.034

Calm J.M., Hourahan G.C., Refrigerant Data Update. HPAC Engineering, 79(1), Page No. 50-64, January-2007. 